\title{
Mechanism of death: there's more to it than sudden cardiac arrest
}

\author{
David C. Parish ${ }^{1}$, Hemant Goyal ${ }^{1}$, Francis C. Dane ${ }^{2}$ \\ ${ }^{1}$ Mercer University School of Medicine, Macon, GA 31201, USA; ${ }^{2}$ Jefferson College of Health Sciences, Roanoke, VA 24013, USA \\ Correspondence to: Hemant Goyal, MD FACP. Assistant Professor of Medicine, Mercer University School of Medicine, 707 Pine St, Macon, GA \\ 31201, USA. Email: doc.hemant@yahoo.com.
}

Submitted Nov 30, 2017. Accepted for publication Apr 12, 2018.

doi: $10.21037 /$ jtd.2018.04.113

View this article at: http://dx.doi.org/10.21037/jtd.2018.04.113

\section{Introduction}

The dying process begins with the loss of function of one or more of the three classic vital organs: heart, brain, lungs. Failure to resuscitate the function of the affected primary organ results in cessation of function of the others. In ventricular fibrillation (VF), for example, the process occurs rapidly as the disorganized activity of the fibrillating heart produces cessation of circulation, which in turn causes loss of consciousness and respiratory drive within seconds. VF, therefore, is primary cardiac arrest; the heart causes the vasculature, brain and lungs to fail. In contrast, pulseless electrical activity (PEA) emerges with collapse of the vascular system, which is a common version of decompensation but rarely discussed in resuscitation literature. The vascular system, therefore, should be viewed as a fourth vital system. Typically, the brain and lungs fail in a sequence that may be so closely linked in time that the first organ to fail is often unclear. As the organism enters death, the heart continues to pump until the oxygen and metabolic substrates required for cardiac function are sufficiently depleted that hypotension and bradycardia emerge, which is followed by loss of an effective pulse. The loss of pulse is the initiation of PEA, but pumping continues when assessed by arterial line or echocardiography (socalled pseudo-PEA) and fades over time (minutes) through PEA to asystole. PEA is not primary cardiac arrest, but is, instead, a late stage in a process of dying that most likely began as arrest of brain, lungs and/or the vascular system. The various patterns of organ failure are delineated and described as mechanisms of dying (Figure 1).

This paper will describe the animal and human studies of the dying process, with and without resuscitative efforts, and delineate the patterns of collapse and time sequence for the reversible portions of dying. We will delineate the primary affected organ system for a variety of insults. Our purpose is to promulgate a more inclusive perception of resuscitation as an attempt to reverse the dying process. The mechanisms of organ failure are separate from the etiology of arrest; as an example, many individuals with decompensated heart failure have the lungs and/or brain arrest before the heart ceases to function.

\section{Models of dying}

Models that distinguish stages of the dying process have been established for over 100 years. It has long been clear that reversal of the process is possible during a brief window of opportunity through artificial maintenance or restoration of the affected vital organ(s). Some have referred to the reversible period following cessation of respiration and pulse as "clinical death" and the irreversible as "biological death". Studies of ECG changes in humans during and after clinical death, for example, began by 1912 (1) shortly after the development of the electrocardiogram (ECG). Clinical observation as the patients declined demonstrated a period of compensation that often included tachycardia, tachypnea, and hypertension followed by a shift in consciousness and decrease in ventilatory drive due to the cessation of function of brain and lungs. After brain and lungs failed, a period of maintained circulatory and cardiac activity included a change in heart rate and mechanism, with a wide range of bradycardic rhythms emerging; blood pressure changed in less than one minute from normal to hypotension and then to pulselessness with loss of electrical cardiac activity over the next several minutes.

Since 1906, animal studies on resuscitation have revealed a similar pattern. The reversible period, which is clinical death, was initiated through a pulmonary or central 
nervous system (CNS) insult. It generally reversed with the elimination of the insult and restoration of breathing during stages with a pulse and blood pressure (2). Once pulselessness occurred, early administration of chest compressions and epinephrine restored circulation. Over a brief period of pulselessness, reversal became less and less likely, then impossible after 10-15 minutes. A wide range of stressors was used in these studies, including hypoxic and anoxic models and rapid bleeding. The initial responses to stressors varied, but the stages from cessation of consciousness and respirations through vascular collapse to asystole were remarkably similar (3-5).

Models of VF in animal studies require induction of VF with shock, which was the initial impetus for the development of the external defibrillator. Kouwenhoven, an electrical engineer at Johns Hopkins, was funded by Continental Edison beginning in the 1920s to develop an external defibrillator capable of reversing VF found in electricity linemen who had received a work-related shock, an uncommon but lethal problem (6). Final development of a functional model by the late 1950s addressed a different and much more significant problem that had emerged during the period in which he was developing the device. The external defibrillator developed by Kouwenhoven was deployed in 1957 by a surgical resident, Dr. Freisinger, to resuscitate a man being prepared for surgery (7).

In 1960 the first resuscitation registry combining rescue breathing, external chest compressions, defibrillation when needed, and cardiotonic drugs, was published (8). Twenty subjects were reported in the series; 14 survived. Only three subjects (two children) were defibrillated. This was the first presentation of the combined package of rescue breathing, chest compressions, adrenergic drugs, and defibrillation in those who needed it. The ability to reverse dying promptly and frequently was transformative. In-hospital resuscitation teams equipped with monitors, defibrillators, ventilators were rapidly established in many hospitals (9). By 1964, trained teams with monitor-defibrillators were placed in public spaces where large groups of people congregated, such as sports stadiums (10). These developments occurred at the peak of the coronary artery epidemic (11) and the patients encountered in these settings had high rates of $\mathrm{VF}$, a complication of undiagnosed or untreated severe coronary heart disease. In studies from 1912 to the 1930s, for example, VF was essentially never seen $(1,12,13)$; studies in the later 30s and 40s demonstrated the emergence of VF in a subset of cases (14). Eventually, VF dominated as the most common rhythm of cardiac arrest in reported literature, especially in pre-hospital groups. Rates of VF as high as $75 \%$ of events were clearly demonstrated in highrisk groups, such as arrest while monitored or witnessed collapse (15). Because of this, research related to VF rapidly expanded. Other rhythms received little empirical or research attention. However, hospital-wide registries generally had lower rates of VF. A review of registries over 30 years had an average rate of $\mathrm{VF}$ of $35 \%$ (16). Our inhospital registry consistently had rates of VF less than $15 \%(17,18)$. Extensive work in public health, improved approaches to diagnosis and treatment of coronary artery disease (CAD), and medical therapy reduced the incidence of $\mathrm{VF}$ as the presenting rhythm in clinical death over the next several decades (19). Currently, PEA is more common than VF in both pre-hospital and in hospital settings $(20,21)$.

\section{PEA}

The first description of PEA in humans is found in an electrocardiographic study of the dying human heart ${ }^{1}$ which was followed by several reports that described the electrocardiographic manifestations of 95 dying patients by the 1930s $(12,13)$. Currently, there is no single unifying available definition for PEA. A report from a National Heart, Lung, and Blood Institute Workshop defined PEA as "a syndrome characterized by the absence of a palpable pulse, in an unconscious patient, with organized electrical activity other than ventricular tachyarrbythmias on ECG." (22). This definition excludes patients with left ventricular assisted devices and patients with agonal, very slow, and wide QRS complexes at the end of prolonged arrest.

Most literature on PEA describes uncertainty regarding how it developed (23); the studies began assessment only during pulselessness, which is deep into the dying process (Figure 1). Infrequent but dramatic causes such as massive pulmonary embolism (PE), cardiac tamponade, and tension pneumothorax have been stressed while more common causes are not discussed. There are several animal models of clinical death that progress to PEA and then asystole; normocarbic hypoxia, hypercarbic hypoxia, anoxia, and rapid bleeding are the most frequently used. Interestingly, none of the animals in these studies entered VF during the process.

Individual models of PEA correspond to common causes of death. Normocarbic hypoxia has been the most frequently studied, as high-altitude loss of consciousness leading to CNS and pulmonary arrest was noted in the early 1900s when balloon and then airplane flight resulted in 


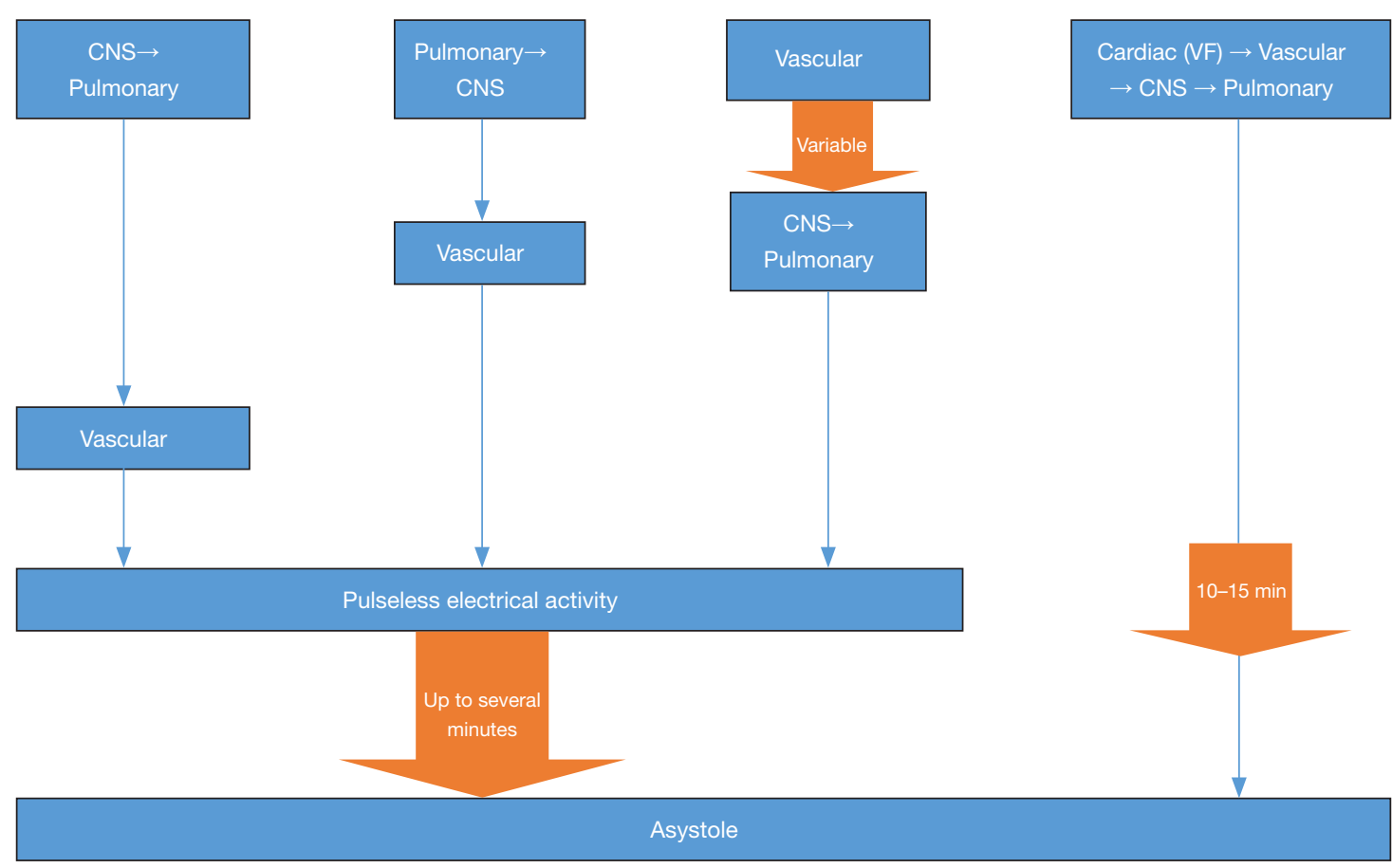

Figure 1 Sequence of organ failure in clinical death. CNS, central south university; VF, ventricular fibrillation.

unexplained deaths. Healthy pilots in normally functioning vehicles crashed with dead pilots. The early experiments with low oxygen developed a chamber which allowed carbon dioxide to be taken out but no oxygen to be added. This was described as a fitness to fly test and variations are still in use. As oxygen levels declined the subjects adapted with deeper respirations but no obvious distress. At a level of hypoxia that was subject specific but ubiquitous, alterations in alertness and cognitive function became apparent, followed shortly by the loss of consciousness and CNS signals for respiration. Provision of normal oxygen resulted in awakening with no awareness of the loss of consciousness $(24,25)$. Animal studies of the same model with continued hypoxia revealed the loss of vascular tone and blood pressure once the residual oxygen was depleted, then PEA developed and proceeded to asystole (26). Disease processes such as pneumonia and congestive heart failure (CHF) commonly follow this trajectory through cognitive dysfunction, loss of respiratory drive, and decay of circulation through PEA to asystole.

Hypercarbic hypoxic models are usually generated by obstruction of the endotracheal tube in a sedated animal (5). Common human correlates of loss of breathing include drug overdose, including excess anesthesia. Pulmonary and CNS arrest from more than safe anesthesia for the individual at that particular point in time provoked more resuscitations in Kouwenhoven's first series than did VF (8). Arrest from imbalance of need for pain control in the perioperative period and patient tolerance of medications over the recovery period continue to be a common cause of such arrests. These are highly survivable if identified by monitors early in the dying process. Other causes include high volume aspiration or foreign body aspiration into the trachea. In the pre-hospital setting, this is the process followed in drowning. The distress caused by airway obstruction or drowning is obvious and dramatic. Failure of consciousness results in collapse, suggesting that brain failure is primary.

Anoxic insults with pure nitrogen provoke rapid CNS and pulmonary arrest; a similar course is followed by the vascular system and heart. While infrequent, the human death equivalent is smoke inhalation or accidental gas exposure in work environments. The inflammatory insult frequently results in death even if the reversal is initiated early in the process.

Bleeding to the point of collapse results in a compensatory tachycardia until the tissue hypoxia is sufficient to cause vascular collapse, followed by CNS then pulmonary collapse. Decompensation of the vascular tone results in bradycardia, PEA, and ultimately asystole. Human 
models similar in progression include trauma, massive gastrointestinal (GI) bleeding, and rupture of a vascular aneurysm. Loss of vascular tone is also the initiating cause of death in anaphylaxis and sepsis. The massive PE described as a saddle embolism stops circulation acutely as venous return, and pulmonary circulation, is entirely blocked. This results in prompt loss of CNS and its dependent respiratory drive.

A common theme in these models is that the brain and lungs often have ceased function before vascular collapse results from global tissue hypoxia as residual oxygen is depleted. Decompensation progresses over a period of minutes even after the pulse is lost. Even when vascular collapse is the primary event, brain and lung functions stops next. The heart is the last organ to fail. The heart stops following PEA, but PEA is not cardiac arrest. The heart finally stops when it arrives at asystole, which is cardiac arrest (Figure 1). The process of decompensation is distinct from the etiology of the collapse; many patients with heart disease as etiology do not currently enter $\mathrm{VF}$ in their dying process. Few patients without heart disease enter VF.

We obtained additional information about PEA through a study of in-hospital resuscitation which began in 1990 by data registry collection on all arrests in one hospital $(7,27)$. The registry contained longitudinal data for 20 years; the emergence of larger data sets and relocation of key team members resulted in discontinuation of the research study. The design included brain and pulmonary failure events from study initiation. Among the positive effects of this choice is that we saw and documented the shift from lung/ brain arrest (1,800 patients) to PEA in about 300 subjects. PEA was the first rhythm identified in over 2,000 subjects. Loss of pulse at any point resulted in similar rates of hospital survival to those first found in PEA. The project stressed standardized definitions and interrater reliability, which allowed us to redefine the eligible subjects and participate in the first study that validated a decision aid for stopping failed resuscitative efforts. The aid had been based on a pulseless subject set (28). While the research registry closed, the first author continues to lead the code team and committee and attends codes regularly as a clinician educator. The hospital has over 600 events yearly, and the patterns described above have not changed. Clinical practice and research in a hospital environment make the continuum of the dying process apparent.

Echocardiographic studies of PEA have established that some subjects have weak cardiac contractions, and others have little or no evidence of wall motion. These have been described as Pseudo-PEA and PEA respectively $(29,30)$. We conducted a prospective, observational study of patients with no history of cardiac disease who were diagnosed with brain death due to intracranial hemorrhage. Institutional review board (IRB) approval was obtained, and families were approached for consent. Three patients were enrolled. These patients were to receive, according to family wishes, hospice/end of life care with no attempt of cardiopulmonary resuscitation or organ harvesting for transplant. Monitoring of blood pressure, oxygen saturation, heart rate and rhythm, and a two-dimensional transthoracic echocardiogram were performed throughout the course of cardiovascular collapse and asystole.

All patients had stable vital signs at the time of disconnection from the ventilator and progressed through PEA to asystole over 12 to 21 minutes, with time to PEA being around 10 minutes. The cardiac function began to decline as oxygen saturation decreased. As the vascular system decompensated, changes in left ventricular ejection fraction (LVEF) and left ventricular internal diameter at diastole (LVIDd) occurred. Diastolic dysfunction was a prominent portion of the collapse in all subjects. PseudoPEA was clearly present at vascular collapse and progressed over minutes to PEA and then asystole (31). Pseudo-PEA and PEA are regular phases in a dying process that can be used to estimate time from collapse.

The decline through PEA to asystole is also observed during organ harvest for transplant, which is begun only at asystole. The strongest evidence that death did not results from failure of the heart or lungs is that transplantation results in normal function of the organ(s), despite a period of asystole (32). Irreversible failure of the lungs is also common; disconnecting ventilatory support from a patient who will never live without it is now an acceptable option for patients and families who choose to stop such interventions.

\section{Resuscitation models}

Two factors dominate the potential for survival of resuscitation: the underlying process that brought the patient to clinical death, and the time in the dying process at which efforts to reverse it, are initiated. If the insult is sufficiently severe, no efforts will be able to reestablish survival with good neurological functioning. The nature and severity of the insult are often not apparent in the acute 
environment, and resuscitative efforts are appropriately begun. VF often occurs abruptly, causing collapse of a previously awake and alert individual. The arrival at clinical death and PEA in other scenarios is a process that can be abrupt (e.g., massive stroke, overwhelming trauma) but more frequently evolves over minutes, hours, or days. Recognition of the time involved and evolution of the dying process has allowed the development of early warning systems and medical emergency teams.

There are four organs and/or systems from which failure can initiate clinical death. The others fail in a clear sequence when the primary insult is not rapidly reversed. Primary cardiac arrest is almost all VF. Primary asystole exists but is sufficiently uncommon to still be reported in the case report literature (33). In all other mechanisms of the dying process, the heart is typically the last organ to fail. In the resuscitation literature there are at least ten different definitions of the level of dysfunction required to be considered a subject of resuscitative efforts, with evidence that survival outcomes are not affected by criterion severity (34). Beginning to look at PEA only after it has become established, rather than as it develops, makes assessment of how it occurred unreliable.

Cessation of brain function may be temporary or permanent; discernment of the magnitude of insult often requires the support of the lungs and sometimes the vascular system to allow clearing of drugs, encephalopathy, or swelling associated with stroke or trauma. PEA and then asystole may occur during this period if ventilatory support is not established in time; resuscitation can still result in a good outcome if the insult is time limited. Ventilatory support can allow the long-term outcome to become clear. Support of lungs will be unneeded as the brain stem function returns and CNS signals restart or support is disconnected for brain death.

\section{Mechanisms of death}

Primary failure of the lungs may be reversed if the insult is removed, for example a foreign body in the trachea, flash pulmonary edema of heart failure resulting in fluid accumulation in the alveoli, or transient ventilator support when due to pneumonia or acute chronic obstructive pulmonary disease (COPD) exacerbation. The dependence of the lungs on CNS stimuli for ventilatory drive is absolute, so failure of the CNS drive during any of these scenarios results in cessation of breathing.
Failure of the vascular system results in tissue hypoxia, altered mentation, and failure of the CNS with the linked cessation of respiratory drive. Substantial work in sepsis and trauma emphasizes the necessity of stabilizing the vascular system to reduce multi-organ failure and irreversible death. Volume repletion is essential and not problematic in these scenarios but will be problematic when the failure is driven by syndromes involving volume overload.

The focus in resuscitation practice and research needs to be shifted to assessment of the primary organ of arrest and the intermediate stages before asystole. PEA is never a primary event and asystole is the final common path for all arrests. Primary cardiac arrest (VF) represents less than $20 \%$ of resuscitation efforts. For research and practice in resuscitation, broadening to include all variants of the dying process will allow collaboration among the many groups working on survival of VF, sepsis, strokes, heart failure, and trauma. We will need a new term for the stages of dying in which reversal is possible, as naming all events sudden cardiac arrest blocks meaningful progress and research. The old term of clinical death may be a candidate, but others will surely emerge. There is considerable potential for progress in an approach that includes all mechanisms of dying in the study of resuscitation. Once we recognize that most deaths do not begin as cardiac arrest, we can begin to develop general resuscitation models.

Interventions to reverse the collapse in trauma, sepsis, CHF, etc., will become part of the spectrum of resuscitation. Therapies initiated at a range of points along the continuum can be assessed for effect on outcome. Profound tissue hypoxia is the underlying cause of arrival at PEA or asystole. All people in PEA and asystole look similar as they are pulseless and apneic, but successful interventions for dehydration, traumatic blood loss, volume overload from end stage renal disease or CHF, or apnea secondary to drug overdose are likely to be different. Markers of the likely etiology in patients found in PEA or asystole can be identified for each mechanism, which could lead to new research questions and interventions.

\section{Acknowledgements}

None.

\section{Footnote}

Conflicts of Interest: The authors have no conflicts of interest 
to declare.

\section{References}

1. Robinson GC. A study with the electrocardiograph of the mode of death of the human heart. J Exp Med 1912;16:291-302.

2. Crile G, Dolley DH. An experimental research into the resuscitation of dogs killed by anesthetics and asphyxia. J Exp Med 1906;8:713-25.

3. Swann HG, Brucer M. The sequence of circulatory, respiratory and cerebral failure during the process of death; its relation to resuscitability. Tex Rep Biol Med 1951;9:180-219.

4. Negovski VA. Agonal states and clinical death; problems in revival of organisms. Am Rev Sov Med 1945;3:147-67.

5. Redding JS, Pearson JW. Resuscitation from asphyxia. JAMA 1962;182:283-6.

6. Beaudouin D. Reviving the body electric. Johns Hopkins Eng 2002;Fall:27-32.

7. Worthington JF. The engineer who could. Hopkins Med News 1998; Winter.

8. Kouwenhoven WB, Jude JR, Knickerbocker GG. Closedchest cardiac massage. JAMA 1960;173:1064-7.

9. McGrath RB. In-house cardiopulmonary resuscitation after a quarter of a century. Ann Emerg Med 1987;16:1365-8.

10. Stokes NA, Scapigliati A, Trammell AR, et al. The effect of the AED and AED programs on survival of individuals, groups and populations. Prehospital Disaster Med 2012;27:419-24.

11. Dalen JE, Alpert JS, Goldberg RJ, et al. The epidemic of the 20th century: coronary heart disease. Am J Med 2014;127:807-12.

12. Turner KB. The mechanism of death of the human heart as recorded in the electrocardiogram. Am Heart J 1931;6:743-57.

13. Hanson JF, Purks WK, Anderson RG.

Electrocardiographic studies of the dying human heart with observations on the intracardiac injection of epinephrine: report of twenty-five cases. Arch Intern Med 1933;51:965-77.

14. Stroud MW, Feil HS. The terminal electrocardiogram: Twenty-three case reports and a review of the literature. Am Heart J 1948;35:910-23.

15. Bayés de Luna A, Coumel P, Leclercq JF. Ambulatory sudden cardiac death: mechanisms of production of fatal arrhythmia on the basis of data from 157 cases. Am Heart J 1989;117:151-9.
16. Schneider AP, Nelson DJ, Brown DD. In-hospital cardiopulmonary resuscitation: a 30-year review. J Am Board Fam Pract 1993;6:91-101.

17. Parish DC, Dane FC, Montgomery M, et al. Resuscitation in the hospital: differential relationships between age and survival across rhythms. Crit Care Med 1999;27:2137-41.

18. Parish DC, Dane FC, Montgomery M, et al. Resuscitation in the hospital: relationship of year and rhythm to outcome. Resuscitation 2000;47:219-29.

19. Parish DC, Dinesh Chandra KM, Dane FC. Success changes the problem: why ventricular fibrillation is declining, why pulseless electrical activity is emerging, and what to do about it. Resuscitation 2003;58:31-5.

20. Tomio J, Nakahara S, Takahashi H, et al. Effectiveness of prehospital epinephrine administration in improving longterm outcomes of witnessed out-of-hospital cardiac arrest patients with initial non-shockable rhythms. Prehosp Emerg Care 2017;21:432-41.

21. Keller SP, Halperin HR. Cardiac arrest: the changing incidence of ventricular fibrillation. Curr Treat Options Cardiovasc Med 2015;17:392.

22. Myerburg RJ, Halperin H, Egan DA, et al. Pulseless electric activity: definition, causes, mechanisms, management, and research priorities for the next decade: report from a national heart, lung, and blood institute workshop. Circulation 2013;128:2532-41.

23. Ornato JP, Peberdy MA. The mystery of bradyasystole during cardiac arrest. Ann Emerg Med 1996;27:576-87.

24. Whitney JL. Cardiovascular observations. J Am Med Assoc 1918;71:1389-91.

25. Gregg HW, Lutz BR, Schneider EC. Compensatory reactions to low oxygen. Am J Physiol. 1919;50:302-26.

26. Greene CW, Gilbert NC. Changes in the pacemaker and in conduction during extreme oxygen as shown in the human electrocardiogram. Arch Intern Med 1921;27:517-57.

27. Jones-Crawford JL, Parish DC, Smith BE, et al. Resuscitation in the hospital: circadian variation of cardiopulmonary arrest. Am J Med 2007;120:158-64.

28. van Walraven C, Forster AJ, Parish DC, et al. Validation of a clinical decision aid to discontinue in-hospital cardiac arrest resuscitations. JAMA 2001;285:1602-6.

29. Paradis NA, Martin GB, Goetting MG, et al. Aortic pressure during human cardiac arrest. Identification of pseudo-electromechanical dissociation. Chest 1992;101:123-8.

30. Salen P, Melniker L, Chooljian C, et al. Does the presence or absence of sonographically identified cardiac activity 
predict resuscitation outcomes of cardiac arrest patients? Am J Emerg Med 2005;23:459-62.

31. Patel RM, Julka K, James E, et al. Abstract 2532: observing dying human heart. Circulation 2009; 120:S655.

32. DeVita MA, Snyder JV, Arnold RM, et al. Observations of withdrawal of life-sustaining treatment from patients who became non-heart-beating organ donors. Crit Care Med 2000;28:1709-12.

Cite this article as: Parish DC, Goyal H, Dane FC. Mechanism of death: there's more to it than sudden cardiac arrest. J Thorac Dis 2018;10(5):3081-3087. doi: 10.21037/ jtd.2018.04.113
33. Zaidan J, Tabet R, Karam B, et al. Asystole caused by Hydroxycut Hardcore: A case report and literature review. Ann Noninvasive Electrocardiol 2017. [Epub ahead of print].

34. Ballew KA, Philbrick JT. Causes of variation in reported in-hospital CPR survival: a critical review. Resuscitation 1995;30:203-15. 